\title{
CARCINOMA IN SITU EX ADENOMA PLEOMORFO EN GLÁNDULA PARÓTIDA, DESCRIPCIÓN DE UN CASO INUSUAL*
}

\author{
Drs. Patricio Cabané T. ${ }^{1}$, Patricio Gac E. ${ }^{1}$, Andrea Muñoz Ch. ${ }^{1}$, Cristina Fernández F. ${ }^{2}$
}

1 Equipo de Cirugía Endocrina-Cabeza y Cuello. Departamento de Cirugía. Hospital Clínico Universidad de Chile.

2 Departamento de Anatomía Patológica. Hospital Clínico Universidad de Chile.

Chile.

\section{Carcinoma ex pleomorphic adenoma}

Objective: To report a case of carcinoma ex pleomorphic adenoma on a young patient. Introduction: Carcinoma ex pleomorphic adenoma typically presents in patients in the sixth decade of life, with a history of parotid tumor of long standing. Alarm symptoms are present in $50 \%$ of cases and survival is mainly correlated with the degree of differentiation and tumor invasion. Case report: A 33 years old woman with right parotid tumor of five years of evolution with slow growing and no symptoms. During the last year, present accelerated growth and stabbing pain. Total parotidectomy was performed without complications or sequelae. The biopsy confirms carcinoma ex pleomorphic adenoma. Discussion: The long-standing pleomorphic adenoma may present malignant transformation in up to $12 \%$. We discuss risk factors, clinical presentation and diagnosis of carcinoma ex pleomorphic adenoma.

Key words: Carcinoma, ex pleomorphic adenoma, parotid tumor.

\section{Resumen}

Objetivo: Presentar un caso clínico de carcinoma ex adenoma pleomorfo en una paciente joven. Introducción: El carcinoma ex adenoma pleomorfo se presenta típicamente en pacientes en la sexta década de la vida, con historia de tumor de parótida de larga data. Tiene síntomas de alarma en el $50 \%$ de los casos y la sobrevida se correlaciona principalmente con el grado de diferenciación y de invasión del tumor. Caso clínico: Paciente de 33 años con tumor de parótida derecho de 5 años de evolución, de lento crecimiento y asintomático. Que durante el último año de evolución presenta dolor punzante y crecimiento acelerado. Se realiza parotidectomía total sin complicaciones ni secuelas. La biopsia confirma un carcinoma ex adenoma pleomorfo. Discusión: El adenoma pleomorfo de larga data puede presentar malignización en hasta un 12\%. Se discute los factores de riesgo, forma de presentación y diagnóstico del carcinoma ex adenoma pleomorfo.

Palabras clave: Adenoma pleomorfo, carcinoma ex adenoma pleomorfo, parotidectomía total.

\footnotetext{
Los autores no refieren conflictos de interés.

Correspondencia: Dr. Patricio Cabané T.

pcabane@hcuch.cl
}

*Recibido el 28 de octubre de 2013 y aceptado para publicación el 14 de febrero de 2014. 


\section{Introducción}

El carcinoma ex adenoma pleomorfo (CXPA) es un tumor maligno de las glándulas salivales mayores $(3,6 \%)$, raro derivado de un adenoma pleomorfo (PA) preexistente ${ }^{1}$. Histológicamente puede dividirse según su extensión en: Carcinoma in situ, carcinoma intracapsular o mínimamente invasivo $(1,5$ $\mathrm{cm}$ o menos de extensión más allá de la cápsula) e invasivo/ampliamente invasivo (mayor que 1,5 $\mathrm{cm})^{2}$. La mayoría de los casos $(81,7 \%)$ se producen en la glándula parótida, mientras que un $18 \%$ en la submandibular y $0,3 \%$ en la glándula sublingual ${ }^{1}$. $\mathrm{Su}$ patogénesis es controversial, pueden ser malignos desde el inicio, o existir una transformación carcinomatosa en el tumor ${ }^{2}$. La transformación en CXPA está relacionada con el tiempo de evolución del adenoma pleomorfo, la edad del paciente y otros factores exógenos como la radiación que se ha puesto en evidencia en pacientes sometidos a radioterapia por tumores de cabeza y cuello donde la probabilidad de desarrollar tumores glandulares malignos se multiplica por cuarenta ${ }^{3}$. La frecuencia de desarrollar malignidad es 1,7 veces mayor en adenoma pleomorfo con características atípicas como hialinización, hipercelularidad, anaplasia celular, necrosis e invasión capsular ${ }^{4}$. El CXPA ocurre generalmente en hombres en la sexta década de la vida y se relaciona con un aumento del riesgo de cáncer pulmonar ${ }^{1,3}$. En fases iniciales, el tumor es indoloro, y la mitad de pacientes no presentan síntomas que sugieren malignidad, como paresia del nervio facial, infiltración de la piel o linfática, dolor o trismus ${ }^{2}$. En tanto, la incidencia de la transformación maligna aumenta desde el $1,6 \%$ de los tumores con un duración clínica de $<5$ años al 9,6\% para los mayores a 15 años $^{5}$. La tasa de supervivencia se ha correlacionado con el tamaño, el tipo y el grado histológico de cáncer; en el carcinoma indiferenciado en adenoma pleomorfo es de $30 \%$ mientras que para los de bajo grado es de $96 \%{ }^{6}$.

\section{Caso clínico}

Paciente de sexo femenino de 33 años de edad, profesora, sin antecedentes mórbidos, presenta aumento de volumen de lento crecimiento retroauricular derecha de $\pm 1 \mathrm{~cm}$ de 5 años de evolución, indolora, sin cambios de coloración en la piel (Figura 1). Se realiza ecotomografía cervical que informa glándula parótida derecha con un nódulo sólido hipoecogénico, bien definido, vascularizado al doppler color de $1,8 \times 1,6 \times 1,7 \mathrm{~cm}$ en sus ejes principales. El nódulo crece $\pm 1 \mathrm{~cm}$ por año, en región infra auricular, evolucionando con dolor punzante intermitente, sensible a la palpación durante el último año, llegando hasta $7 \mathrm{~cm}$ de diámetro aproximadamente, circunscrito, de consistencia dura, móvil y piel de aspecto normal. Se realiza ecotomografía cervical que informa en glándula parótida derecha una masa hipoecogénica, heterogénea de contornos lobulados bien definida de aproximadamente $4 \times 2,9 \times 2,5 \mathrm{~cm}$, en sus ejes longitudinal, anteroposterior y transversal respectivamente, que podria corresponder a un adenoma pleomorfo que ha aumentado de tamaño respecto al nódulo del estudio anterior (Figura 2). El tratamiento

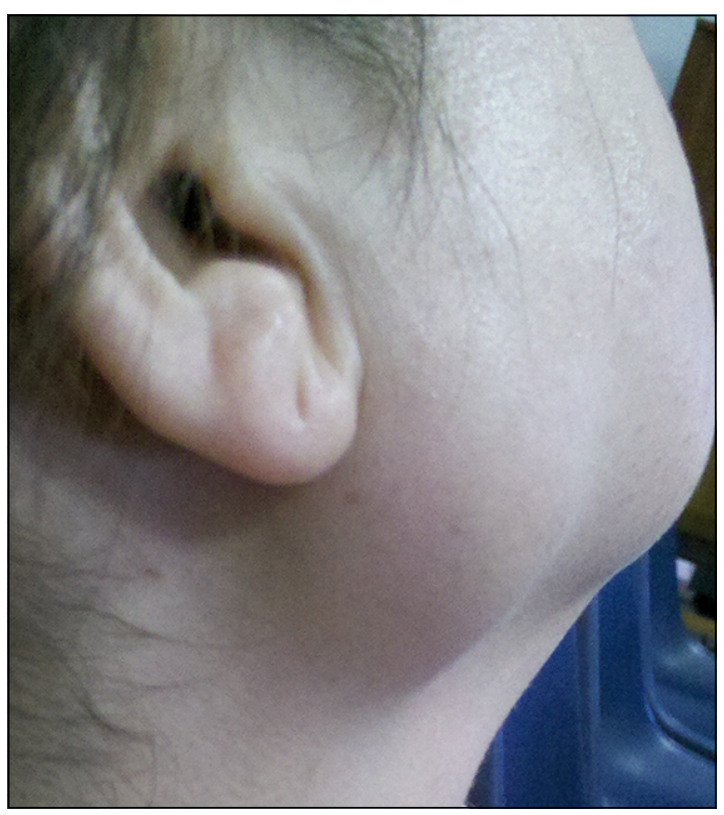

Figura 1. Masa de $7 \mathrm{~cm}$ aproximadamente, sin cambios de coloración de la piel. En región infraauricular.

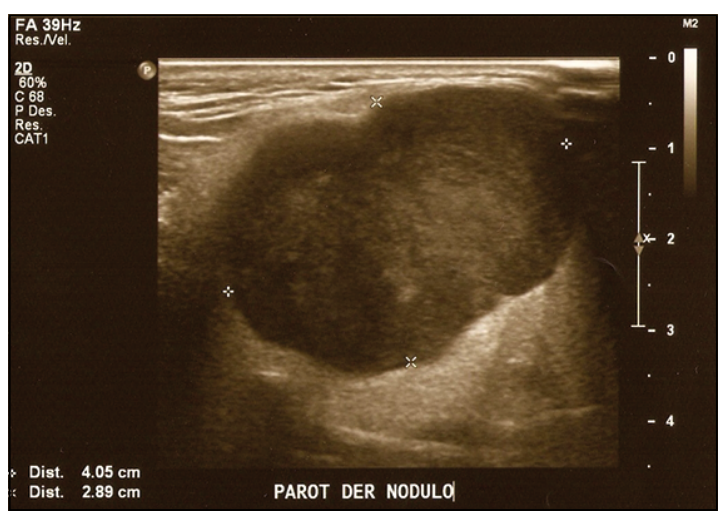

Figura 2. Glándula parótida derecha con una masa hipoecogénica, heterogénea de contornos lobulados bien definida de aproximadamente 4 × 2,9, en sus ejes longitudinal y anteroposterior respectivamente. 

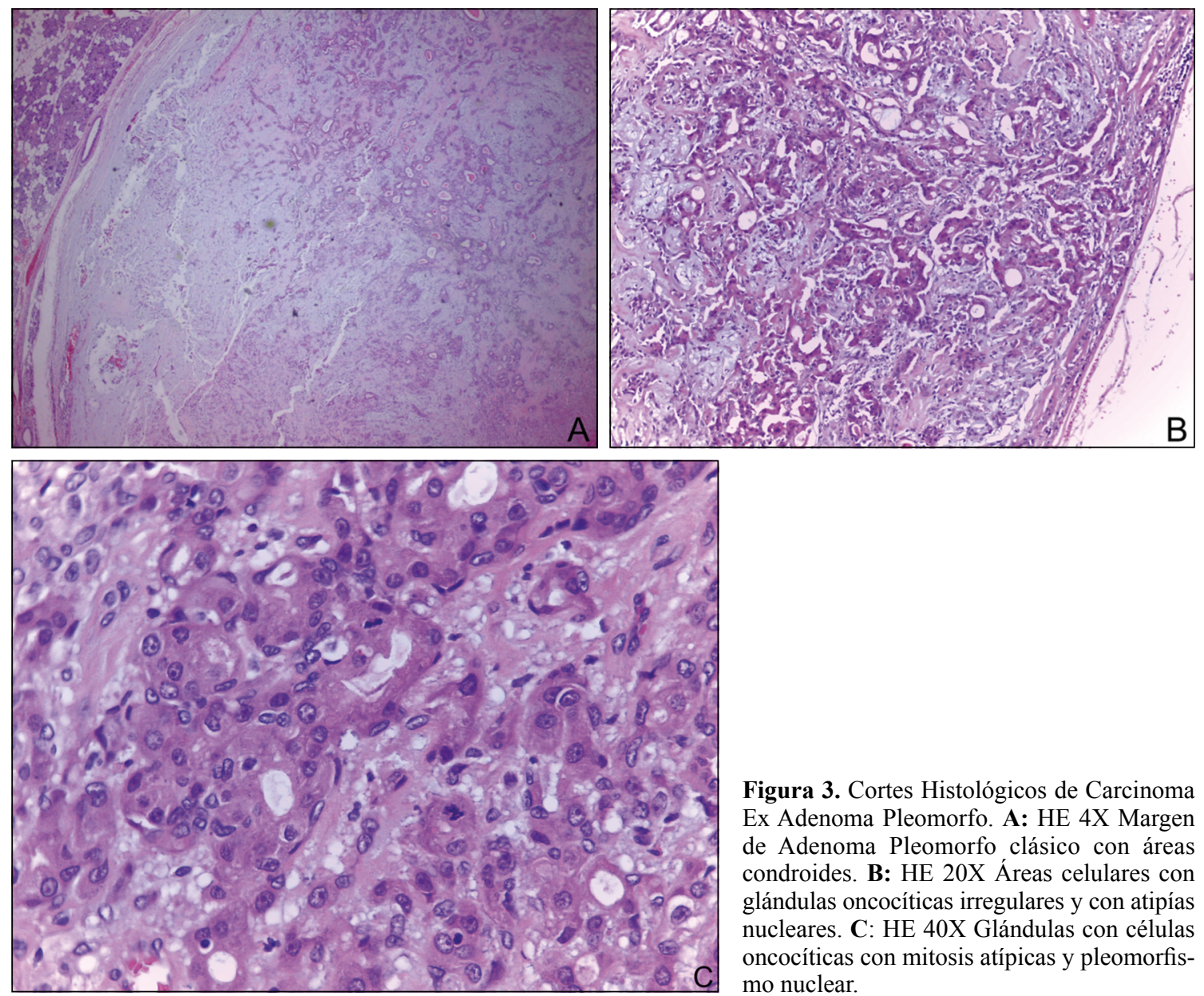

Figura 3. Cortes Histológicos de Carcinoma Ex Adenoma Pleomorfo. A: HE 4X Margen de Adenoma Pleomorfo clásico con áreas condroides. B: HE 20X Áreas celulares con glándulas oncocíticas irregulares y con atipías nucleares. C: HE 40X Glándulas con células oncocíticas con mitosis atípicas y pleomorfismo nuclear.

fue Parotidectomía total Derecha, por parámetros preoperatorios e informe de biopsia contemporánea con sospecha de carcinoma. No se realizó disección ganglionar por no tener evidencia clínica ni radiológica de adenopatías cervicales. La paciente en su postoperatorio evoluciona de manera satisfactoria, función del VII par craneano normal, sin evidencias de fístula salival ni hematoma. El tumor resecado fue informado en biopsia definitiva como un carcinoma in situ en ex adenoma pleomorfo, intracapsular, de 4 x $3 \mathrm{~cm}$, sin compromiso capsular. Tinción citoplasmática cerb-b2 leve en la zona tumoral y Ki67 en menos del 1\% (Figura 3). Evoluciona asintomática, sin evidencias de recidiva. Actualmente, la paciente permanece en control semestral.

\section{Discusión}

El CXPA es un tumor maligno englobado en el epitelio de un PA. Su origen puede ser un adenoma pleomorfo no tratado y malignizado (el intervalo de malignización puede ser de hasta 20 años) o una malignización de una recurrencia tumoral ${ }^{7}$. Se presenta en el 8,8\% de los tumores mixto y en el $4,6 \%$ de los tumores de glándulas salivales malignos ${ }^{8}$. El CXPA ocurre generalmente en hombres en la sexta década de la vida ${ }^{1}$. En este caso clínico el tumor se desarrolló de manera temprana a los 33 años de vida en una mujer, inusual a lo descrito en la literatura, aunque en el estudio de Zbären y cols. ${ }^{2}$ también tuvo predominio femenino pero la edad media fue de 67 años. Los signos sospechosos de malignidad son dolor, la parálisis facial y adenopatías, que se presentan en aproximadamente el $30 \%$ de los pacientes y cambios de coloración y textura de la piel circundante en menor medida ${ }^{2}$. El CXPA da metástasis a ganglios linfáticos, huesos (especialmente cuerpos vertebrales), y al cerebro. Las metástasis a distancia son más frecuentes que las regionales ${ }^{1}$. Hasta un $60-70 \%$ de los pacientes desarrollan metástasis local 
o distantes ${ }^{9}$. El diagnóstico preoperatorio se basa en la historia clínica, el examen físico, estudios de imagen y citología por punción aspirativa con aguja fina (PAAF). El dolor, empastamiento, alteraciones neurológicas y/o cutáneas o la clínica de reciente comienzo, tras años de evolución de una masa parótida asintomática alertan de esta patología. La ecografía es un estudio simple y de bajo costo que permite evaluar las glándulas salivales y las cadenas ganglionares cervicales. Actualmente, la técnica de imagen más empleada es la Tomografía Computada (TC), con una sensibilidad de $64-92 \%$ y especificidad del 86-98\%; que analiza la lesión tumoral, la extensión loco-regional y a distancia. La resonancia magnética, tiene igual utilidad que la TC. La PAAF detecta malignidad con una especificidad de 86 a $98 \%$; la sensibilidad, 64 a 92\%, y su objetivo es diferenciar entre tumores benignos, malignos y lesiones inflamatorias, siendo limitada su utilidad por la escasa muestra que se examina ${ }^{3}$. El diagnóstico definitivo de CXPA se define con el estudio histológico de la pieza quirúrgica, y es requisito para el diagnóstico la detección de adenoma pleomorfo en asociación a focos de carcinoma. Es útil para el diagnóstico también el alto índice proliferativo medido por un anticuerpo monoclonal denominado Ki-67 y la positividad del protooncogén Her-2 /neu (cerbB-2) $)^{4}$ que puede ser un marcador útil para identificar la transformación maligna de PA. Otros marcadores que pueden ayudar a detectar el cambio carcinomatoso temprano, son: PCNA y p53. En el caso descrito se encontró positividad para cerbB-2 y un mínimo de $1 \%$ de positividad para Ki67.

El tratamiento de elección es quirúrgico y la técnica varía según aspectos clínicos y radiológicos del tumor. Si no afecta el nervio facial se realizará parotidectomía total conservadora, y en los que presentan paresia de alguna de las ramas, se realizará parotidectomía radical con resección del nervio. La disección cervical funcional es clara en aquellos casos clínicamente positivos. Actualmente, el vaciamiento supraomohioideo es el tratamiento profiláctico de la enfermedad metastásica oculta. Algunos autores no recomiendan el vaciado cervical programado como tratamiento estándar, ya que los carcinomas de glándula salivar mayor metastatizan más frecuentemente por vía hematógena que linfática y raramente metastatizan en los ganglios cervica$\mathrm{les}^{3}$. El tratamiento adyuvante con radioterapia tiene muy buenos resultados en cuanto a supervivencia y curación. Sus indicaciones incluyen: márgenes quirúrgicos afectados o muy próximos, extensión extraglandular (T3 o T4), invasión perineural y presencia de adenopatías cervicales o intraparotídeas ${ }^{3,10}$. El papel de la quimioterapia postoperatoria adicional para CXPA no es claro, pero hay que considerar el estado general, la edad, el estadio tumoral y detalles histológicos. El pronóstico está dado por múltiples factores: el tamaño de la invasión tumoral, el tipo histológico y la presencia de metástasis loco regionales o a distancia ${ }^{3}$. Aunque el subtipo histológico tiene cierta relevancia, el indicador pronóstico más importante es el grado de invasión en el adenoma pleomorfo (PA). Tortoledo y cols encontró que en carcinomas que invadieron $<6 \mathrm{~mm}$ todos tenían un resultado favorable, pero que aquellos que invadieron $>8 \mathrm{~mm}$ siempre el resultado era fatal ${ }^{5}$. La tasa de recurrencia del adenoma pleomorfo varía de un $4-45 \%$ y también depende de la técnica quirúrgica empleada (parotidectomía vs tumorectomía) ${ }^{7}$. La transformación maligna puede llegar hasta un $12 \%$.

\section{Referencias}

1. Mag A, Cotulbea S, Marin A, Doros C, Neamtu D, Balica N, et al. Carcinoma ex pleomorphic adenoma in parotid gland- case report, Journal of Experimental Medical \& Surgical Research. 2010;17:205-9.

2. Zbären P, Zbären S, Caversaccio M, Stauffer E. Carcinoma ex pleomorphic adenoma: Diagnostic difficulty and outcome, Otolaryngology, Head and Neck Surgery 2008;138:601.

3. Rodríguez M, García M, Rodríguez M, Herrera G, Fernández M, García B, y cols. Carcinoma ex adenoma parotídeo: a propósito de un caso. Revista Española Cirugía Oral Maxilofacial 2010;32:125-7.

4. Logasundaram R, Hellquist H, Amarawickrama H, Premachandra D. Intracapsular (in situ) carcinoma ex pleomorphic adenoma with unusual clinical and histological features, Eur Arch Otorhinolaryngol 2008;265:1563-6.

5. Di Palma S, Skálová A, Vanieek T, Simpson R, Starek I, Leivo I. Non-invasive (intracapsular) carcinoma ex pleomorphic adenoma: recognition of focal carcinoma by HER-2/neu and MIB1 immunohistochemistry, Histopathology 2005;46:144-52.

6. Rodríguez J, Micas M, Martínez F, Berjón J, Montalvo J, Forteza G, et al. Metastatic benign pleomorphic adenoma. Report of a case and review of the literature. Med Oral Patol Oral Cir Bucal 2008;13:E193-6.

7. Navarro I, González J, Muñoz JM, Pingarrón L, Del Castillo JL, Benito DM, y cols. Carcinoma ductal sobre adenoma pleomorfo de parótida, Revista Española Cirugía Oral y Máxilofacial 2009;31:397-402.

8. Ellis G, Auclir P. Tumors of the salivary glands. Whashington, DC: Armed Forces Institute of Pathology. 1996 Atlas of tumors Pathology, 3.

9. Tawfik O, Namiq A. Tumors of the Salivary Glands, Cancer Grading Manual, 2007, pp 13-8.

10. Lüers J, Wittekindt C, Streppel M, Guntinas-Lichius O. Carcinoma ex pleomorphic adenoma of the parotid gland. Study and implications for diagnostics and therapy 2009;48:132-6. 